\title{
ATENDIMENTO INTEGRAL E COMUNITÁRIO EM SAÚDE MENTAL: AVANÇOS E DESAFIOS DA REFORMA PSIQUIÁTRICA
}

\author{
COMPREHENSIVE AND COMMUNAL USER EMBRACEMENT IN MENTAL HEALTH: \\ PROGRESSES AND CHALLENGES OF THE PSYCHIATRIC REFORM
}

\begin{abstract}
Francisco de Sales Clementino iD (hhttps://orcid.org/0000-0001-8470-4694) ${ }^{1}$, Francisco Arnoldo Nunes de Miranda ID (https://orcid.org/0000-0002-8648-811X)², João Mário Pessoa Júnior iD) (hhttps://orcid.org/0000-0003-2458-6643) ${ }^{3}$ Emanuella de Castro Marcolino iD (hhttps://orcid.org/0000-0002-6135-8853)², José Antônio da Silva Júnior (iD) (https://orcid.org/0000-0001-7965-3095) ${ }^{1}$, Gisetti Corina Gomes Brandão iD (https://orcid.org/0000-0001-8040-5435) ${ }^{1}$
\end{abstract}

\author{
${ }^{1}$ Universidade Federal de Campina Grande, Centro de Ciências Biológicas e da Saúde, Unidade \\ Acadêmica de Enfermagem, Campina Grande, Paraíba, Brasil. \\ $<$ fclementino67@yahoo.com.br> \\ ${ }^{2}$ Universidade Federal do Rio Grande do Norte, Centro de Ciências da Saúde, Departamento de \\ Enfermagem, Natal, Rio Grande do Norte, Brasil. \\ ${ }^{3}$ Universidade Federal Rural do Semi-Árido, Centro de Ciências Biológicas e da Saúde, Departamento de \\ Ciências da Saúde, Mossoró, Rio Grande do Norte, Brasil.
}

\begin{abstract}
Resumo Objetivou-se caracterizar e analisar a organização dos processos de trabalho em serviços de saúde mental no município de Campina Grande, Paraíba, Brasil. Trata-se de um estudo quantitativo, retrospectivo e documental, realizado entre julho e agosto de 2014. Coletaram-se os dados a partir de formulário baseado em instrumento validado para o estudo, considerando uma amostra de 402 prontuários. Para o processamento das análises estatísticas, utilizou-se o SPSS, versão 17.0. Observou-se um perfil de usuários predominantemente composto por mulheres na faixa etária adulta, com diagnóstico clínico de esquizofrenia, associado a outros diagnósticos clínicos. Quanto às internações em hospitais psiquiátricos antes e após o ingresso no serviço, notou-se redução estatisticamente significante. Como importante avanço, evidenciaram-se os elevados registros de oficinas realizadas. Como principal limitação do estudo, apontam-se falhas nos registros dos prontuários, mas que não os tornam menores ou irrelevantes. Reforça-se que o atendimento integral e de base comunitária ao usuário com transtorno mental e sua família, condizente com os preceitos reformistas, requer, entre outros, maiores investimentos na infraestrutura dos serviços, equipamentos e tecnologias, apoio institucional, capacitação e comprometimento da gestão nos processos de educação permanente.
\end{abstract}

Palavras-chave serviços de saúde mental; recursos humanos; saúde mental.
Abstract The goal of the article was to characterize and analyze the organization of the work processes in mental health services in the municipality of Campina Grande, in the state of Paraíba, Brazil. It is a quantitative, retrospective and documentary study that was performed between July and August 2014. The data was collected from a form based on an instrument validated for the study, considering a sample composed of 402 medical records. In order to process the statistical analyses, we used the SPSS software, version 17.0. We observed that the users were predominantly adult women with a clinical diagnosis of schizophrenia associated with other clinical diagnoses. Regarding the admissions to psychiatric hospitals before and after enrolling in the service, we noticed a statistically significant reduction. The high number in the records of workshops held was an important progress. The main limitations of the study were the mistakes in the medical records, which do not make them less important or irrelevant, though. We stress that the comprehensive and communal embracement of the users with mental disorders and their families, which is in line with the psychiatric reform norms, requires, among other things, higher investments in the infrastructure of the services, equipment and technologies, institutional support, qualification and commitment of the management in the ongoing education processes. Keywords mental health services; human resources; mental health.

(cc) BY Este é um artigo publicado em acesso aberto sob uma licença Creative Commons. 


\section{Introdução}

No Brasil, a Reforma Psiquiátrica encerra um ciclo do protagonismo do hospital psiquiátrico e seu modelo hospitalocêntrico hegemônico com a extinção progressiva dos leitos psiquiátricos, na medida em que redesenha um novo modelo de atenção psicossocial. A lei federal n. 10.216/2001 (Brasil, 2001) redireciona e assegura às pessoas com transtornos mentais a assistência em saúde mental cujo tratamento é ofertado em serviços de base comunitária (Guedes et al., 2014). Somam-se outros dispositivos e estratégias no campo da saúde mental implementados, sem, contudo, perder de vista o fio condutor da desinstitucionalização e do reforço ao cuidado integral de base territorial e humanizado (Brasil, 2015; Macedo et al., 2017).

Com os avanços e desafios reformistas, emergiu a Rede de Atenção Psicossocial (RAPS) instituída pelo decreto n. 7.508/2011 que a define pelos seguintes serviços: Centros de Atenção Psicossocial (CAPSs), Serviços Hospitalares de Referência, os Serviços Residenciais Terapêuticos (SRTs), os Centros de Convivência de Cultura e Consultórios de Rua, dentre outros (Brasil, 2015). O trabalho multiprofissional e intersetorial é valorizado e considerado como estratégico no processo de articulação e comunicação entre os espaços e equipamentos pertencentes ao território (Santos, 2014; Pessoa Júnior et al., 2016).

No transcurso de mudanças e transformações sociais, econômicas e políticas emergiram desafios no campo da saúde mental e atenção psicossocial, especialmente no que tange à organização dos serviços e ao processo de trabalho entre as equipes. Tem-se também a falta de investimentos financeiros para instalação e funcionamento satisfatório dos serviços, bem como ações em saúde fragmentadas e desarticuladas, com práticas centradas no modelo biomédico (Dutra e Oliveira, 2015; Zeferino et al., 2016).

Observam-se, ainda, dificuldades na gestão com reduzido quantitativo de serviços, equipamentos e recursos humanos, bem como as reinternações frequentes na atenção terciária (Coelho et al, 2014; Pessoa Júnior et al., 2016). Em contrapartida, o processo de regionalização da RAPS permitiu a ampliação do acesso, cobertura e melhoria da qualidade de atenção em saúde mental em todos os níveis e pontos de atenção no Sistema Único de Saúde (SUS). Apesar dessa capilarização, destacam-se os 'vazios assistenciais' em diversos pontos de atenção, fragilizando o atendimento entre os serviços (Macedo et al, 2017).

No âmbito das políticas públicas nacionais, a etapa de consolidação da desinstitucionalização e do trabalho em rede exige, além da substituição dos hospitais por serviços comunitários, um olhar crítico para o processo de trabalho dos profissionais sob o risco de que reproduzam nos locais de trabalho o modelo manicomial e segregador prestado em unidades asilares de outrora (Bessa e Waidman, 2013). 
O presente estudo tem, portanto, como pergunta principal: Como se caracteriza e se organiza o processo de trabalho em serviços de saúde mental? Tem-se como objetivo caracterizar e analisar a organização dos processos de trabalho em serviços de saúde mental no município de Campina Grande, Paraíba, Brasil.

\section{Método}

Estudo quantitativo, retrospectivo e documental realizado em Campina Grande, Paraíba, nos cinco Centros de Atenção Psicossocial (CAPSs), no período de julho a agosto de 2014. Desses serviços, dois são classificados como tipo I (localizados nos distritos sanitários de Galante e São José da Mata); um, do tipo II e um, do tipo III, além de um CAPS Álcool e Drogas (AD), totalizando 2.297 prontuários. Destes, calculou-se uma amostra aleatória estratificada com partilha proporcional. Consideraram-se os seguintes parâmetros: erro $\alpha$ de $5 \%$, nível de confiança de $95 \%$, poder do estudo de $80 \%$, estimativa de proporção de $50 \%$ e índice de proporcionalidade específico, perfazendo 330 prontuários.

Do quantitativo definido, foram acrescentados $25 \%$ de prontuários para eventuais perdas. Respeitado esse acréscimo, a amostra final foi composta de 413 prontuários, assim distribuídos: CAPS I - São José da Mata ( $\mathrm{n}=49 ; 11,9 \%)$; CAPS I - Galante ( $\mathrm{n}=40 ; 9,7 \%)$; CAPS II - Novos Tempos $(\mathrm{n}=85 ; 20,6 \%)$; CAPS III - Reviver ( $\mathrm{n}=170 ; 41,2 \%)$; CAPS AD - $(\mathrm{n}=69 ; 16,6 \%)$. Ressalta-se que os prontuários incluídos são de: maiores de 18 anos, de ambos os sexos, com registro de admissão no CAPS há pelo menos seis meses e que não estivessem sem atendimento por igual período. Excluíram-se, portanto, os prontuários dos usuários que receberam alta, bem como daqueles que foram transferidos para outros serviços de saúde, além daqueles com mais de seis meses ausentes do serviço ofertado pela RAPS.

Com a aplicação de um questionário validado e desenvolvido para o estudo Capsul (2007), coletaram-se os dados a partir de dois grupos de variáveis. As primeiras são relativas aos usuários, tais como sexo (masculino/feminino); faixa etária (adultos: 20 a 59 anos/idosos: 60 anos ou mais); diagnóstico; data de ingresso no serviço; internações psiquiátricas em hospitais gerais e psiquiátricos anteriores e posteriores ao ingresso no CAPS. As outras variáveis são relativas ao cuidado oferecido, tais como: procedência do usuário; profissional responsável pela primeira avaliação; modalidade de acompanhamento atual; registro de atendimento, intercorrências, visitas domiciliares, atendimento/orientação aos familiares; caracterização do Plano Terapêutico Individual (PTI) - registro, espaço de definição, participação de profissionais, itens que o constituem -; última história clínica (sinais e sintomas, história familiar de transtorno mental, uso de medicação, funções psíquicas, apoio 
familiar, adesão às atividades, perfil socioeconômico); psicofármacos em uso e indicados na última prescrição.

Inicialmente, realizou-se análise descritiva. Para avaliar o impacto da implantação do CAPS sobre as internações hospitalares, aplicou-se o teste do quiquadrado de Fisher. A comparação do tipo de diagnóstico por sexo foi realizada pelo teste de Bonferroni ajustado. Em toda a análise, adotou-se o nível de significância de 5\%, utilizando-se o SPSS 17.0.

Seguiram-se os requisitos da Resolução n. 466/12 do Conselho Nacional de Saúde. O estudo foi aprovado pelo Comitê de Ética em Pesquisa da Universidade Federal do Rio Grande do Norte, protocolo n. 719.435, em 30/05/2014.

\section{Resultados e discussão}

O perfil e a densidade populacionais das cidades constituem um dos principais critérios para o planejamento da rede de atenção à saúde mental dos municípios e para a implementação dos serviços especializados. As ações de saúde devem ser pautadas com base na população a qual elas servirão. Nesse sentido, convém destacar que dados censitários últimos afirmam que 89\% dos municípios do território nacional apresentam densidade populacional de até 50.000 habitantes. Desse modo, são considerados municípios de pequeno porte (Brasil, 2015).

\section{Caracterização dos usuários}

Foram registradas 11 perdas por falha ou ausência de preenchimento dos prontuários, perfazendo uma amostra final de 402 registros avaliados. A maioria referia-se a usuários do sexo feminino $(\mathrm{n}=207 ; 51,5 \%)$ e de faixa etária adulta $(\mathrm{n}=329 ; 81,8 \%)$. Observaram-se apenas 48 idosos $(11,9 \%)$ e, em 25 prontuários (6,2\%), não havia registro da idade (dados não tabulados).

Corroborando os resultados desta pesquisa, estudos de perfil epidemiológico em CAPSs apresentaram predominância de usuários do sexo feminino (Miranda, Oliveira e Santos, 2014; Oliveira et al., 2014). Embora o percentual de idosos acometidos tenha sido inferior ao de adultos, o dado é preocupante, sobretudo porque distúrbios psíquicos em idosos costumam ocorrer devido a diferentes fatores psicossociais, como a vivência de perdas significativas, enfraquecimento da vida social e sofrimentos advindos da desvalorização da pessoa, maus tratos, isolamento e solidão, dentre outros (Cavalcante et al., 2015). Além disso, a longevidade aproxima a mais temida das perdas: a da própria vida (Souza et al., 2013). 


\section{Caracterização dos diagnósticos}

Quando avaliados os diagnósticos que levaram à busca pelo CAPS, verificou-se que quase $60 \%$ concentraram-se em apenas um grupo da CID-10: F20-F29 (esquizofrenia, transtornos esquizotípicos e delirantes). O menor percentual $(0,3 \%)$ foi registrado para 'Síndromes comportamentais associadas a distúrbios fisiológicos e fatores físicos' (F50-F59), com igual frequência para o 'Transtorno mental não especificado' (F-99). Um total de 77 diagnósticos não estava registrado nos prontuários. Verificou-se diferença estatisticamente significante na distribuição por sexo para os diagnósticos de F10-F19, F30F39 e F40-F48. Do total de diagnósticos, dois acometem, prioritariamente, as mulheres e um, os homens (Tabela 1). O mesmo não foi verificado para a faixa etária (dados analisados, mas não apresentados).

Tabela 1

Distribuição dos diagnósticos recebidos pelos usuários avaliados nos cinco Centros de Atenção Psicossocial, segundo o sexo. Campina Grande, Paraíba, 2002-2014.

\begin{tabular}{|c|c|c|c|c|c|c|c|}
\hline Diagnóstico & Descrição & \multicolumn{2}{|c|}{$\begin{array}{c}\text { Total } \\
(n=402)\end{array}$} & \multicolumn{2}{|c|}{$\begin{array}{l}\text { Masculino } \\
(n=195)\end{array}$} & \multicolumn{2}{|c|}{$\begin{array}{l}\text { Feminino } \\
(\mathrm{n}=207)\end{array}$} \\
\hline F10-F19 & $\begin{array}{l}\text { Transtornos mentais e comportamentais } \\
\text { relativos ao uso de álcool, múltiplas } \\
\text { drogas e outras substâncias psicoativas }\end{array}$ & 51 & 12,7 & $45 a$ & 88,2 & $06_{b}$ & 11,8 \\
\hline F20-F29 & $\begin{array}{l}\text { Esquizofrenia, transtornos esquizotípicos } \\
\text { e delirantes }\end{array}$ & 194 & 48,3 & $91_{a}$ & 46,9 & 103 a & 53,1 \\
\hline F30-F39 & Transtornos de humor (afetivos) & 35 & 8,7 & $11_{a}$ & 31,4 & $24_{b}$ & 68,6 \\
\hline F40-F48 & $\begin{array}{l}\text { Transtornos neuróticos relacionados com } \\
\text { o estresse e somatoformes }\end{array}$ & 26 & 6,5 & 05 & 19,2 & $21_{b}$ & 80,8 \\
\hline F50-F59 & $\begin{array}{l}\text { Síndromes comportamentais associadas } \\
\text { com distúrbios fisiológicos e fatores } \\
\text { físicos }\end{array}$ & 01 & 0,3 & 00 & - & $01_{\mathrm{a}}$ & 100,0 \\
\hline F70-F79 & Retardo mental & 13 & 3,2 & $08_{a}$ & 61,5 & $05_{a}$ & 38,5 \\
\hline F80-F89 & $\begin{array}{l}\text { Transtornos do desenvolvimento } \\
\text { psicológico }\end{array}$ & 04 & 1,0 & $01_{a}$ & 25,0 & 03 & 75,0 \\
\hline F99 & Transtorno mental não especificado & 01 & 0,3 & $01_{a}$ & 50,0 & $00_{a}$ & 50,0 \\
\hline- & Diagnóstico não registrado no prontuário & 77 & 19,0 & $33 a$ & 42,9 & $44 a$ & 57,1 \\
\hline
\end{tabular}

As colunas identificadas por 'a, b' são aquelas em que foram identificadas diferenças significativas dos diagnósticos entre os sexos. Teste de Bonferroni ( $p$ ajustado $<0,05$ )

Fonte: Os autores.

Em estudo realizado em um serviço de saúde mental, em São João DelRei - MG, observou-se resultado semelhante. Dentre os transtornos mentais apresentados, a maioria referia-se à esquizofrenia, seguida por transtorno afetivo bipolar; outros transtornos psicóticos não orgânicos; transtorno esquizotípico; e outros transtornos mentais (Bonadiman, Bonadiman e Silva, 2013). 
Pesquisa realizada em um CAPS II de Santarém-PA (Soares et al., 2015) constatou predominância de mulheres entre 31 e 40 anos e os transtornos mentais mais prevalentes foram episódio depressivo, esquizofrenia e transtorno afetivo bipolar. Tal constatação pode estar associada à exposição a situações difíceis relativas às responsabilidades domésticas, baixos salários, discriminações no mercado de trabalho e outras aflições da vida social. Estas informações foram consideradas para a elaboração do Plano de Ação para a Saúde Mental 2013-2017.

\section{$O$ atendimento profissional}

Do total dos 402 prontuários avaliados, constatou-se, em 2002, o primeiro registro em uma unidade de CAPS, um ano após ter sido instituída a Lei da Reforma Psiquiátrica. Após esse marco inicial no município do estudo, verificou-se, até 2009, o cadastramento de apenas 119 usuários $(29,6 \%)$ nos CAPSs existentes em Campina Grande. Posteriormente a 2010, um ano marcado pela IV Conferência Nacional de Saúde Mental, resultante da Marcha pela Reforma Psiquiátrica em 2009, o número de usuários cadastrados aumentou, chegando, até setembro de 2014, a $275(68,4 \%)$, um incremento superior ao dobro em um período inferior a quatro anos. Ressalta-se que, diante das falhas de preenchimento dos prontuários do total de cinco CAPSs, não foi possível verificar a data de ingresso de oito usuários $(2 \%)$.

Com relação às internações hospitalares, verifica-se um comportamento inverso. O número total reduziu significativamente; da mesma forma, antes do cadastro em uma unidade de CAPS, pelo menos 14 (3,5\%) usuários haviam sido internados em hospital geral e 117 (29,1), em hospital psiquiátrico. Após o acompanhamento nos serviços substitutivos, as internações reduziram para $7(1,7 \%)$ e $11(2,7 \%)$, respectivamente, sendo esta última estatisticamente significante (Tabela 2).

\section{Tabela 2}

Frequência de internações hospitalares antes e após o ingresso dos usuários avaliados nos Centros de Atenção Psicossocial. Campina Grande e região, Paraíba, 2002-2014.

\begin{tabular}{|c|c|c|c|c|c|}
\hline \multirow[t]{2}{*}{ Internações } & \multicolumn{2}{|c|}{ Antes do CAPS } & \multicolumn{2}{|c|}{ Após o CAPS } & \multirow{2}{*}{$\mathbf{p}$} \\
\hline & $\mathbf{n}$ & $\%$ & $\mathbf{n}$ & $\%$ & \\
\hline \multicolumn{6}{|l|}{ Internações Gerais } \\
\hline Sim & 131 & 32,6 & 18 & 4,5 & \multirow{3}{*}{0,001} \\
\hline Não & 271 & 67,4 & 384 & 95,5 & \\
\hline Total & 402 & 100,0 & 402 & 100,0 & \\
\hline \multicolumn{6}{|l|}{ Hospital Geral } \\
\hline Uma & 9 & 64,3 & 6 & 85,7 & \multirow{3}{*}{$0,612^{*}$} \\
\hline Duas ou mais & 5 & 35,7 & 1 & 14,3 & \\
\hline Total & 14 & 100,0 & 7 & 100,0 & \\
\hline \multicolumn{6}{|l|}{ Hospital Psiquiátrico } \\
\hline Uma & 39 & 33,3 & 9 & 81,8 & \multirow{3}{*}{$0,002^{*}$} \\
\hline Duas ou mais & 78 & 66,7 & 2 & 18,2 & \\
\hline Total & 117 & 100,0 & 11 & 100,0 & \\
\hline
\end{tabular}


No âmbito da Política Nacional de Saúde Mental e em reforço ao processo de desinstitucionalização, recomenda-se que as internações psiquiátricas hospitalares, quando necessárias, sejam realizadas em hospitais gerais. Dados ministeriais apontam que, até 2014, havia 4.620 leitos de psiquiatria em hospitais gerais, pediátricos e maternidades destinadas ao SUS. Em dezembro de 2014, o Brasil contava com 116 municípios-sede de hospitais psiquiátricos e 1.488 municípios com CAPSs em seus territórios. Tal fato evidencia a capilaridade destes centros, bem como a realização concreta da transformação do modelo centrado nos hospitais psiquiátricos para o modelo de atenção psicossocial de base comunitária (Brasil, 2015).

Mesmo diante da implementação dos CAPSs entre os municípios brasileiros, especialmente nas modalidades I e II, o número de serviços comunitários com funcionamento 24 horas (CAPS III) e os voltados aos cuidados de populações específicas, como o CAPS infantil e CAPS álcool e drogas, ainda é bastante reduzido (Coelho et al., 2014).

Estudo realizado em hospitais psiquiátricos na cidade de Belo Horizonte (MG) identificou uma alteração no perfil de atendimento no período compreendido entre 2002 e 2011 , com redução dos atendimentos externos, aumento do número de internações de menor duração, redução relativa dos atendimentos de oriundos da própria capital e predominância de diagnósticos de transtornos ligados ao uso de álcool e drogas (Coelho et al., 2014). Concluiu-se que a implantação de comunitários tenha retirado o hospital do foco da assistência a essa população, embora se tenha um número considerável de usuários com internações de curta duração. Ademais, a extinção total dos hospitais psiquiátricos requer, entre outros pontos, maiores investimentos de ordem política, econômica e social com vistas a estimular o processo de ampliação e de estruturação de serviços que compõem a Rede de Atenção Psicossocial.

\section{O fluxo na rede de atenção à saúde mental entre os CAPSs}

Em relação aos dados demonstrativos do fluxo da rede de atenção à saúde mental do município em estudo, no que tange à procedência do usuário cadastrado nos CAPSs, constata-se que, para quase metade dos usuários, 199 $(49,5 \%)$, o encaminhamento ao CAPS ocorreu por meio de vizinhos e familiares. As unidades básicas de saúde apareceram em segundo lugar, com 76 usuários encaminhados (18,9\%); seguidas pelas emergências, com 44 casos (10,9\%); o hospital geral, com 17 (4,2\%); os hospitais psiquiátricos, com 14 (3,5\%); e o ambulatório especializado, com 13 casos (3,3\%). Para 39 usuários $(9,7 \%)$, essa informação estava ausente.

Em consonância com os resultados do estudo, pesquisa realizada no Ceará mostrou que 48,6\% dos usuários atendidos pelos CAPSs foram encaminhados pelo hospital público mental, o que mostra que o modelo tradicional de hos- 
pital ainda predomina quando se trata de saúde mental (Costa et al., 2015). Nesse sentido, conhecendo a importância do funcionamento dessa rede no manejo, as situações de crise revelam inúmeras experiências vividas pelos usuários que mostram como a acessibilidade se configura, tanto para garantir o benefício do cuidado necessário quanto para apontar possibilidades de barreiras efetivas ao acesso que deseja (Gomes e Merhy, 2014).

Dentre os profissionais de saúde de nível superior que compõem as equipes dos CAPSs, o psicólogo foi apontado como o profissional que mais participou do primeiro atendimento, em 80 casos (19,9\%), seguido dos assistentes sociais $(\mathrm{n}=57 ; 14,2 \%)$. No tocante às visitas domiciliares (VD) registradas para o mês anterior à coleta, o registro foi muito baixo, com apenas dez registros. $\mathrm{O}$ assistente social foi o profissional que mais vezes as realizaram, ainda que em uma quantidade pequena, seguido pelos enfermeiros e técnicos de enfermagem, que fizeram três visitas cada, e psicólogos, com apenas uma. Não houve registro de visitas domiciliares para a categoria médica.

Constata-se, no presente estudo, que a realização da VD pelos profissionais de saúde mostra-se ineficaz, dada a sua insuficiência e irregularidade, o não envolvimento de todos profissionais nesta atividade social e terapêutica, especialmente o profissional médico. Nesse sentido, alega-se aumento da demanda de atendimento, equipe reduzida e falta de transporte, dentre outros. No contexto da Saúde Mental, a VD possibilita manter o paciente afastado da internação psiquiátrica e aparece como cuidado da equipe, o qual preconiza o acolhimento e a troca de experiências, pois a casa do paciente fornece pistas e dados para que haja algum nexo entre a doença e o social (Fernandes, Duarte e Schmalfuss, 2014).

Estudo realizado em Natal-RN (Santos, 2014) mostrou que os profissionais informaram que os atendimentos domiciliares se encontravam prejudicados por razões operacionais e de logística, como a falta de transporte, uma vez que existia apenas um veículo disponível para todas as modalidades de CAPSs no município. Neste contexto, a visita domiciliar pode não ser efetiva ou ocorrer sem a devida frequência pela equipe multiprofissional dos serviços de saúde (Fernandes, Duarte e Schmalfuss, 2014).

\section{O plano terapêutico individual}

Avaliaram-se aspectos relativos ao plano terapêutico individual (PTI), desde a implantação até as características de funcionamento. A maioria dos usuários ainda não tem atendimento com registro de PTI (n=214; 53,2\%); mais de $80 \%$ dos CAPSs não dispõem de um espaço de atuação do PTI. No período estudado, chamam a atenção os elevados registros de realização de oficinas $(\mathrm{n}=133)$, atendimento individual $(\mathrm{n}=109)$ e prescrição de fármacos 
$(\mathrm{n}=122)$. Em contrapartida, há um reduzido número de incentivos ao Programa de Geração de Renda (n=3) (Tabela 3).

Tabela 3

Caracterização do plano terapêutico individual de 188 usuários cadastrados nas unidades de Centro de Atenção Psicossocial. Campina Grande, Paraíba, 2002-2014.

\begin{tabular}{|c|c|c|}
\hline Espaço de definição do plano terapêutico individual & $\mathbf{n}$ & $\%$ \\
\hline \multicolumn{3}{|l|}{ Em acolhimento } \\
\hline Sim & 51 & 12,7 \\
\hline Não & 347 & 86,3 \\
\hline Sem registro & 4 & 1,0 \\
\hline \multicolumn{3}{|l|}{ Em consulta individual } \\
\hline Sim & 75 & 18,7 \\
\hline Não & 323 & 80,3 \\
\hline Sem registro & 4 & 1,0 \\
\hline \multicolumn{3}{|l|}{ Em reunião de equipe } \\
\hline Sim & 68 & 16,9 \\
\hline Não & 329 & 81,8 \\
\hline Sem registro & 5 & 1,2 \\
\hline \multicolumn{3}{|l|}{ Em outro espaço } \\
\hline Sim & 15 & 3,7 \\
\hline Não & 380 & 94,5 \\
\hline Sem registro & 7 & 1,7 \\
\hline \multicolumn{3}{|l|}{ Participação de profissionais na definição do PTI } \\
\hline Assistente social & 43 & 10,7 \\
\hline Enfermeiro & 54 & 13,4 \\
\hline Médico & 97 & 24,1 \\
\hline Psicólogo & 97 & 24,1 \\
\hline Outro profissional & 64 & 15,9 \\
\hline Sem registro & 47 & 11,8 \\
\hline \multicolumn{3}{|l|}{ Presença de itens que compõem o PTI* } \\
\hline Inserção no trabalho & 14 & - \\
\hline Programa de geração de renda & 3 & - \\
\hline Inserção comunitária & 47 & - \\
\hline Cuidados pessoais com o corpo & 15 & - \\
\hline Grupo de conversação & 86 & - \\
\hline Oficinas & 133 & - \\
\hline Acompanhamento aos familiares & 75 & - \\
\hline Atendimento individual & 109 & - \\
\hline Prescrição de fármacos & 122 & - \\
\hline Visita domiciliar & 19 & - \\
\hline
\end{tabular}

Nota: PTI - plano terapêutico individual; *A presença de itens que compõem o PTI pode conter mais de uma resposta Fonte: Os autores.

Pesquisa realizada em dois CAPSs da região metropolitana de Fortaleza-CE mostrou entraves para a realização do PTI (transposição da barreira da fragmentação; insuficiente participação dos usuários e seus familiares; dificuldade de ampliação das ações para além do CAPS) (Vasconcelos et al., 2016). Este fato favorece a produção de ações isoladas em que o acolhimento, a escuta do sujeito, a humanização e a integralidade, por vezes, são pouco explorados pela equipe. Além disso, mostra a omissão de propostas claras e estrategicamente definidas para os usuários em sofrimento psíquico, no bojo das políticas locais da atenção básica em saúde.

É importante entender que o PTI não é um protocolo a ser preenchido, mas uma estratégia para a melhoria da atenção psicossocial, baseada em um modelo de escuta, diálogo, decisão conjunta, compartilhamento do poder e participação ativa (Rodrigues e Deschamps, 2016). Reforça-se a necessidade do cuidado qualificado em saúde mental e do diálogo intersetorial no conjunto de ações desenvolvidas nessa área como capaz de contribuir no processo 
de reinserção social e familiar dos usuários com transtornos mentais (Pessoa Júnior et al., 2016).

No campo das políticas públicas em saúde mental, há obstáculos a serem superados, como, por exemplo, o subfinanciamento no SUS para a implementação da rede substitutiva, a qual centraliza o modelo nos CAPSs, com pouco incentivo aos outros componentes da rede de cuidados. Outros entraves são as dificuldades para inserção da atenção básica na rede de cuidados em saúde mental e a insuficiência da criação de serviços com funcionamento 24 horas, dentre outros (Lobosque, 2011).

Somam-se, às condições de trabalho insatisfatórias, o desrespeito aos anseios dos trabalhadores, como também a visão meramente tecnicista e burocrática; desqualificam o cuidado e, por conseguinte, a humanização das práticas (Jorge et al, 2015; Santos, 2016). Além disso, observa-se a ausência de uma política de recursos humanos em saúde mental que estimule a qualificação e valorização dos profissionais atuantes na área.

\section{A clínica e assistência aos usuários}

No tocante à última avaliação clínica, 338 usuários apresentam registro de manifestação de algum sinal e/ou sintoma (84\%). Verificou-se história familiar de transtorno mental para $282(70,1 \%)$ usuários; uso de medicação por 346 (86\%); avaliação de funções psíquicas em 222 (55,2\%); apoio familiar para $204(50,7 \%)$; adesão às atividades propostas pela equipe por $204(50,7 \%)$ (dados não tabulados).

Quanto ao uso de medicamentos, os principais grupos de psicofármacos em uso e indicados na última prescrição incluem: antipsicóticos e neurolépticos $(40,5 \%)$; seguidos por anticonvulsivantes (18,9\%); ansiolíticos e hipnóticos $(14,7 \%)$; inibidores seletivos da receptação de serotonina $(9,7 \%)$; outros antidepressivos (1,2\%); e de outros grupos, como antiparkisonianos, analgésicos não opioides e para doença de Alzheimer (16,2\%).

Estudo desenvolvido no Centro de Assistência Psicossocial de FortalezaCeará indica o alto uso de medicamentos psicotrópicos, principalmente antipsicóticos, anti-histamínicos e antidepressivos (Costa et al., 2015). O que se vê é uma tendência, ainda predominante, de enfrentamento de doença, também observada no Programa de Melhoria do Acesso e da Qualidade da Atenção Básica (PMAQ-AB), que mostrou que as ações de promoção de saúde voltadas para a "Prevenção e tratamento ao uso, abuso e dependência de ansiolíticos e benzodiazepínicos" se mostram como atividades menos produzidas (Clementino et al., 2014).

Assim, entende-se que a predominância da racionalidade instrumental/ técnica e a fragilidade dos mecanismos de regulação do trabalho em saúde mental impactam na quantidade e qualidade do acesso e utilização dos recur- 
sos de saúde, na capacidade resolutiva das equipes, no cuidado continuado e na atuação no território (Macedo et al, 2017).

\section{Conclusão}

Observou-se um perfil de usuários predominantemente composto por mulheres na faixa etária adulta, com diagnóstico clínico de esquizofrenia e também associado a outros diagnósticos clínicos. Quanto às internações em hospitais psiquiátricos antes e após o ingresso nos CAPS, notou-se redução estatisticamente significante. E, no que tange ao processo de trabalho, foram evidenciados como importantes avanços os elevados registros de realização de oficinas realizadas no CAPS que constituem, entre outros, novas formas de acolhimento, de convivência e trocas de experiências. Entre os entraves, destacam-se a ausência de registro do atendimento dos usuários acompanhados no projeto terapêutico individual, bem como a irregularidade da visita domiciliar, que ocorre de forma esporádica. Essa situação ocorre, tendo em vista obstáculos como aumento da demanda de atendimento, equipe reduzida e falta de transporte.

Chama-se a atenção para a necessidade de repensar os processos de trabalho por parte da gestão e das equipes de profissionais, no sentido de fortalecer o atendimento na RAPS, em especial no serviço substitutivo analisado, considerando seu papel estratégico neste cenário. Desse modo, é notório o reflexo direto do modelo biomédico, o qual compreende a doença mental apenas do ponto de vista orgânico. Reforça-se que o atendimento integral e de base comunitária ao usuário com transtorno mental e à sua família, de forma condizente com os preceitos reformistas e de base comunitária, requer maiores investimentos. Estes aportes devem ser empregados na infraestrutura dos serviços, equipamentos e tecnologias, apoio institucional, capacitação e comprometimento da gestão nos processos de Educação Permanente em Saúde, entre outros.

Reconhecem-se, como principal limitação do estudo, falhas nos registros dos prontuários, seja pela ausência de determinadas informações seja pelo preenchimento incorreto de dados importantes sobre o usuário, utilizandose, unicamente, de dados secundários, mas que não os tornam menores ou irrelevantes.

Em contrapartida, há um esforço e uma intenção de acrescentar ao campo da atenção psicossocial elementos capazes de adensar o processo de regionalização e constituição das redes de atenção à saúde no Brasil. Desse modo, é possível, após caracterização, organizar o sentido para futuras investigações e, quiçá, para ações de planejamento de políticas em saúde mental. 


\section{Colaboradores}

Francisco de Sales Clementino foi responsável pela concepção, elaboração, redação e revisão final do texto. Francisco Arnoldo Nunes de Miranda colaborou na elaboração, redação e revisão crítica do texto. João Mário Pessoa Júnior colaborou na redação e revisão final do texto. Emanuella de Castro Marcolino colaborou na revisão crítica do texto. José Antônio da Silva Júnior e Gisetti Corina Gomes Brandão colaboraram na revisão final do texto.

\section{Financiamento}

Não houve financiamento.

\section{ATENCIÓN INTEGRAL Y COMUNITARIA EN SALUD MENTAL: AVANCES Y DESAFÍOS DE LA REFORMA PSIQUIÁTRICA}

Resumen Se buscó caracterizar y analizar la organización de los procesos de trabajo en servicios de salud mental en el municipio de Campina Grande, Paraíba, Brasil. Se trata de un estudio cuantitativo, retrospectivo y documental, realizado entre julio y agosto del 2014. Se recolectaron los datos a partir de un formulario basado en el instrumento validado para el estudio, considerando una muestra de 402 historias clínicas. Para el procesamiento de los análisis estadísticos se utilizó el SPSS, versión 17.0. Se observó un perfil de usuarios predominantemente compuesto por mujeres de edad adulta, con diagnóstico clínico de esquizofrenia, asociado a otros diagnósticos clínicos. Respecto a las internaciones en hospitales psiquiátricos antes y después del ingreso al servicio, se observó una reducción estadísticamente significativa. Como avance importante se destacan los altos registros de talleres realizados. Como principal limitación del estudio se indican fallas en los registros de las historias clínicas, pero que no las hacen menos importantes o irrelevantes. Se hace énfasis en que la atención integral y de base comunitaria al usuario con trastorno mental y a su familia, de acuerdo con las políticas reformistas, requiere entre otros aspectos de mayores inversiones en la infraestructura de los servicios, equipos y tecnologías, apoyo institucional, capacitación y compromiso de la gestión en los procesos de educación permanente.

Palabras clave servicios de salud mental; recursos humanos; salud mental. 


\section{Referências}

BESSA, Jacqueline B.; WAIDMAN, Maria A. P. Família da pessoa com transtorno mental e suas necessidades na assistência psiquiátrica. Texto \& Contexto em Enfermagem, Florianópolis, v. 22, n. 1, p. 61-70, 2013.

BONADIMAN, Samuel L.; BONADIMAN, Raphael L.; SILVA, Denise A. Avaliação do uso do biperideno em pacientes sob tratamento com fármacos antipsicóticos. Acta Biomedica Brasiliensia, Itaperuna, RJ, v. 4, n. 1, 2013. Disponível em: <http://www.actabiomedica. com.br/index.php/acta/article/view/51>. Acesso em: 13 nov. 2014.

BRASIL. Lei n. 10.216/2001, de 06 de abril de 2001. Dispõe sobre a proteção e os direitos das pessoas portadoras de transtornos mentais e redireciona o modelo assistencial em saúde mental. Diário Oficial da República Federativa do Brasil, Poder Executivo, Brasília, DF, 09 abr. 2001. Disponível em: <http://www. planalto.gov.br/ccivIl_03/LEIS/LEIS_2001/ L10216.htm>. Acesso em: 20 set. 2016.

BRASIL. Lei n. 7.508 de 28 jun. 2011. Regulamenta a Lei $\mathrm{n}^{\mathrm{o}} 8.080$, de 19 de setembro de 1990, para dispor sobre a organização do Sistema Único de Saúde - SUS, o planejamento da saúde, a assistência à saúde e a articulação interfederativa, e dá outras providências. Diário Oficial da República Federativa do Brasil, Poder Executivo, Brasília, DF, 29 jun. 2011. Disponível em: <http://www.planalto.gov. br/ccivil_03/_ato2011-2014/2011/decreto/ d7508.htm > . Acesso em: 20 set. 2016.

BRASIL. Ministério da Saúde. Coordenação Geral de Saúde Mental. Saúde mental em dados 12, 2015. 48p. Disponível em: < http://www. mhinnovation.net/sites/default/files/downloads/innovation/reports/Report_12-edicao-doSaude-Mental-em-Dados.pdf > . Acesso em: 15 set. 2017.

CAPSUL. Avaliação dos CAPS da Região Sul do Brasil: estudo qualitativo. Coordenação Luciane Prado Kantorski. Pelotas: Ministério da Saúde, 2007. p. 437. Disponível em: <http:// www2.ufpel.edu.br/feo/capsul/estudo \% 20 qualitativo.pdf > . Acesso em: 16 abr. 2017.

CAVAlCANTE, Ana C. S. et al. A clínica do idoso em situação de vulnerabilidade e risco de suicídio. Revista Trivium Estudos Interdisciplinares, Rio de Janeiro, v. 7, n. 1, p. 74-87, 2015.

CLEMENTINO, Francisco S. et al. Ações de promoção da saúde: uma análise a partir do Programa de Melhoria do Acesso e da Qualidade da Atenção Básica (AB). In: SEMINÁRIO INTERNACIONAL DE PROMOÇÃO DA SAÚDE, 5., 2014, Fortaleza. Anais... Fortaleza, 2014.

COELHO, Vivian A. A. et al. Alteração do perfil de atendimento dos hospitais psiquiátricos públicos de Belo Horizonte, Brasil, no contexto da reforma da assistência à saúde mental. Ciência \& Saúde Coletiva, Rio de Janeiro, v. 19, n. 8, p. 3.605-3.616, 2014.

COSTA, Rosiana C. et al. Perfil epidemiológico de usuários intensivos de um Centro de Atenção Psicossocial. Revista de Enfermagem da UFPE, Recife, v. 9, n. 2, p. 820-829, 2015.

DUTRA, Virginia F. D.; OLIVEIRA, Rosane M. P. Revisão integrativa: as práticas territoriais de cuidado em saúde mental. Aquichán, Chía, v. 15, n. 4, p. 529-540, 2015.

FERNANDES, Marivana; DUARTE, Maria L. C.; SCHMALFUSS, J. M. Facilidades e dificuldades na realização de visitas domiciliares em um Centro de Atenção Psicossocial. Cogitare Enfermagem, Curitiba, v. 19, n. 3, p. 451-458, 2014.

GOMES, Maria P. C.; MERHY, Emerson E. (org.). Pesquisadores IN-MUNDO: um estudo da produção do acesso e barreira em saúde mental. Porto Alegre: Rede Unida, 2014.

GUEDES, Ariane C. et al. Tratamento em liberdade: pontos de tensionamento e avanço no Centro de Atenção Psicossocial. Revista de Enfermagem da UFSM, Santa Maria, v. 4, n. 1, p. 133-43, 2014. 
JORGE, Maria S. B. et al. Apoio matricial, projeto terapêutico singular e produção do cuidado em saúde mental. Texto \& Contexto em Enfermagem, Florianópolis, v. 24, n. 1, p. 112-120, 2015.

LOBOSQUE, Ana M. Debatendo alguns desafios da reforma psiquiátrica brasileira. Ciência \& Saúde Coletiva, Rio de Janeiro, v. 16, n. 12, p. 4.590-4.592, 2011.

MACEDO, João P. et al. A regionalização da saúde mental e os novos desafios da Reforma Psiquiátrica brasileira. Saúde e Sociedade, São Paulo, v. 26, n. 1, p. 155-170, 2017.

MIRANDA, Lilian; OLIVEIRA, Thaíssa. F. K.; SANTOS, Cátia B. T. Estudo de uma Rede de Atenção Psicossocial: paradoxos e efeitos de precariedade. Psicologia: Ciência e Profissão, Brasília, v. 34, n. 3, p. 592-611, 2014. Disponível em: <http://www.scielo.br/pdf/ pcp/v34n3/1982-3703-pcp-34-03-0592.pdf >. Acesso em: 19 set. 2016.

OLIVEIRA, Valdimeire F. et al. Caracterização de pacientes com transtornos mentais atendidos no Centro de Atenção Psicossocial em São Francisco do Conde - Bahia. Revista de Ciências Médicas e Biológicas, Salvador, v. 13, n. 2, p. 204-211, 2014. Disponível em: < https:// portalseer.ufba.br/index.php/cmbio/article/ view/11672/9070>. Acesso em: 29 set. 2016.

PESSOA JÚNIOR, João M. et al. A política de saúde mental no contexto do hospital psiquiátrico: desafios e perspectivas. Escola Anna Nery Revista de Enfermagem, Rio de Janeiro, v. 20, n. 1, p. 83-89, 2016.
RODRIGUES, Jeferson; DESCHAMPS, Andréa L. P. Política de saúde mental e projeto terapêutico singular. Cadernos Brasileiros de Saúde Mental, Florianópolis, v. 8, n. 17, p. 78-92, 2016.

SANTOS, Raionara C. A. Papéis e funções dos profissionais dos serviços e politicas de saúde mental em Natal (RN). 2014. 89f. Tese (Doutorado em Enfermagem)- Faculdade de Enfermagem, Universidade Federal do Rio Grande do Norte, Natal, 2014.

SANTOS, Iraci. Tecnologias do cuidar destinadas a diminuir as disparidades em saúde mental. Revista Portuguesa de Enfermagem de Saúde Mental, Porto, n. especial 4, p. 6-8, 2016.

SOARES, Anna C. et al. Análise clínica-epidemiológica de pacientes portadores de transtorno mental na Amazônia brasileira. Revista Brasileira de Neurologia e Psiquiatria, Salvador, v. 19, n. 2, p. 96-107, 2015.

SOUZA, Andréa S. et al. Perfil sociodemográfico e de saúde de idosos com sintomas depressivos. Revista de Enfermagem da UERJ, Rio de Janeiro, v. 21, n. 3, p. 355-360, 2013.

VASCONCELOS, Mardênia G. F. et al. Projeto terapêutico em Saúde Mental: práticas e processos nas dimensões constituintes da atenção psicossocial. Interface- Comunicação, Saúde, Educação, Botucatu, v. 20, n. 57, p. 313-323, 2016

ZEFERINO, Maria T. et al. Percepção dos trabalhadores da saúde sobre o cuidado às crises na Rede de Atenção Psicossocial. Escola Anna Nery Revista de Enfermagem, Rio de Janeiro, v. 20, n. 3, jul. /set. 2016 . 